\title{
Exploring Potential Proteomic Biomarkers for Prognosis of Infective Endocarditis through Profiled Autoantibodies by an Immunomics Protein Array Technique
}

\author{
Chang-Hua Chen,,${ }^{1,2,3,4}$ Ying-Cheng Chen, ${ }^{5}$ Ching-Hui Huang, ${ }^{6,7,8}$ Shu-Hui Wang, ${ }^{9}$ Jen-Shiou Lin, ${ }^{10}$ \\ Shou-Chen Lo, ${ }^{11}$ Chieh-Chen Huang ${ }^{3,11,12}$
}

\begin{abstract}
${ }^{1}$ Division of Infectious Diseases, Department of Internal Medicine, Changhua Christian Hospital, Changhua, Taiwan; ${ }^{2}$ Center for Infection Prevention and Control, Changhua Christian Hospital, Changhua, Taiwan; ${ }^{3}$ Ph.D. Program in Translational Medicine, National Chung Hsing University, Taichung County, Taiwan; ${ }^{4}$ Rong Hsing Research Center For Translational Medicine, National Chung Hsing University, Taichung County, Taiwan; ${ }^{5}$ Division of Cardiovascular Surgery, Changhua Christian Hospital, Taiwan; ${ }^{6}$ Division of Cardiology, Department of Internal Medicine, Changhua Christian Hospital, Changhua, Taiwan; ${ }^{7}$ Department of Beauty Science, Graduate Institute of Beauty Science Technology, Chienkuo Technology University, Changhua, Taiwan; ${ }^{8}$ School of Medicine, College of Medicine, Kaohsiung Medical University, Kaohsiung, Taiwan; ${ }^{9}$ Division of Critical Care Medicine, Changhua Christian Hospital, Changhua, Taiwan; ${ }^{10}$ Department of Laboratory Medicine, Changhua Christian Hospital, Changhua, Taiwan; ${ }^{11}$ Department of Life Sciences, National Chung Hsing University, Taichung County, Taiwan; ${ }^{12} \mathrm{Ph} . \mathrm{D}$. Program in Medical Biotechnology, National Chung Hsing University, Taichung County, Taiwan
\end{abstract}

\section{ABSTRACT}

Though infective endocarditis (IE) is a life-threatening cardiac infection with a high mortality rate, the effective diagnostic and prognostic biomarkers for IE are still lacking. The aim of this study was to explore the potential applicable proteomic biomarkers for IE through the Immunome ${ }^{\mathrm{TM}}$ Protein Array system. The system was employed to profile those autoantibodies in IE patients and control subjects. Our results showed that interleukin-1 alpha (IL1A), nucleolar protein 4 (NOL4), tudor and $\mathrm{KH}$ domain-containing protein (TDRKH), G antigen 2B/2C (GAGE2), glyceraldehyde-3-phosphate dehydrogenase $(G A P D H)$, and $\mathrm{X}$ antigen family member 2 (XAGE2) are highly differentially-expressed among IE and non-IE control. Furthermore, bactericidal permeability-increasing protein $(B P I)$, drebrin-like protein $(D B N L)$, signal transducing adapter molecule 2 (STAM2), cyclin-dependent kinase 16 (CDK16), BAG family molecular chaperone regulator 4 (BAG4), and nuclear receptor-interacting protein 3 (NRIP3) are differentially-expressed among IE and healthy controls. On the other hand, those previously identified biomarkers for IE, including erythrocyte sedimentation rate, C-reactive protein, rheumatoid factor, procalcitonin, and $\mathrm{N}$-terminalpro-B-type natriuretic peptide demonstrated only minor significance. With scientific rationalities for those highly differentially-expressed proteins, they could serve as potential candidates for diagnostic biomarkers of IE for further analysis.

Received April 16, 2020; accepted June 10, 2020.

Correspondence: Chieh-Chen Huang, Ph.D., Department of Life Sciences, National Chung Hsing University., No. 250 Kuo-Kuang Road, Taichung 402, Taiwan (e-mail: cchuang@dragon.nchu.edu.tw); Chang-Hua Chen, M.D., M.Sc., Ph.D., Division of Infectious Diseases, Department of Internal Medicine, Changhua Christian Hospital, Changhua, Taiwan. No 135 Nanshia Street, Changhua 500, Taiwan (e-mail: changhua@cch.org.tw).

\section{INTRODUCTION}

Infective endocarditis (IE) is a life-threatening cardiac infection accompanied by the destroyed cardiac valves and leading to heart failure [Beynon 2006; Habib 2009; Thiene 2006]. Further, vegetations can induce the embolism and damage the brain and other organs. Although there is improvement with earlier diagnosis and more intensive therapeutic strategies, the mortality rate for IE still is $15-40 \%$ [Knudsen 2009; Habib 2015; Wei 2017]. As a matter of fact, though early identification of IE patients is known to be critical to make accurate clinical decisions and improve patient prognosis, the mean delay of the diagnostic period for IE is reported to be approximately 30 days [Knudsen 2009]. In our previous studies, some critical neurologic complications caused by endocarditis were found in central Taiwan [Chen 2001; Chen 2001; Chen 1997], while the in-hospital mortality rate of IE was $29.3 \%$ [Chen 2001]. Further, the rate of neurologic complications was $24 \%$, and the adjusted

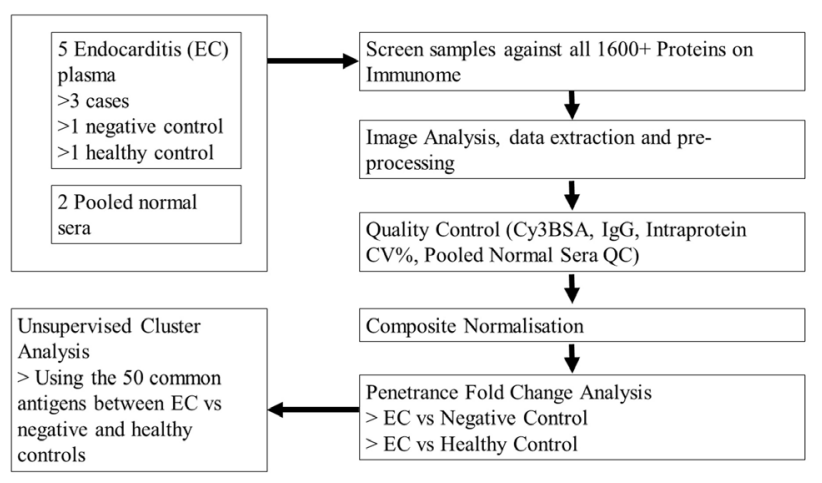

Figure 1. Study protocol 

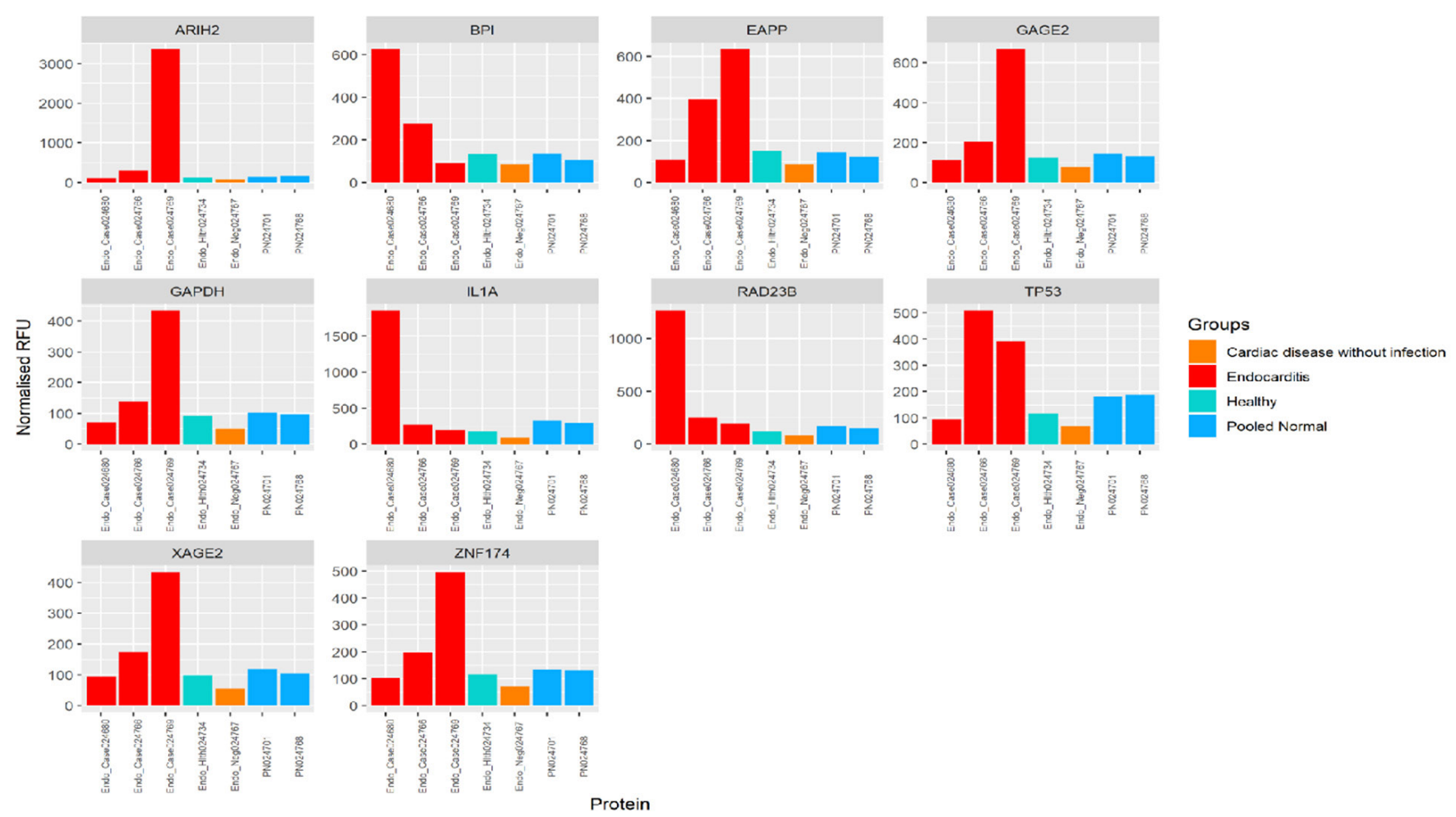

Figure 2. Autoantibody profile across IE, non-IE control, healthy control and pooled normal samples for the most clinically significant common biomarkers identified based on penetrance fold change analysis between IE vs. non-IE control and IE vs. healthy control. Legend: Endo_Case024680 , Endo_Case024766 and Endo_Case024769 are 3 IE patient samples; Endo_Health024734 is 1 healthy control sample; Endo_Neg024767 is 1 non-IE patient sample; PN024701 and PNO24768 are 2 pooled normal sera.

risk ratio for neurologic complications for a fatal event was 3.51 (95\% confidence interval, $1.10-11.18, P=.03$ ) [Chen 2001].

The development of biomarkers with high predictive value for infectious diseases is one of the goals of precision medicine. Early diagnosis, patient stratification, and predictive treatment of infectious diseases using biomarkers can improve treatment effectiveness and significantly enhance the survival rate of patients, especially in cases of acute and difficult infectious diseases [Chen 2001; Chen 2001; Li 2000]. The factors that may determine the neurologic complications and their impacts for IE patients are important issues that need to be revealed. Therefore, how to distinguish IE from fever patients with bacteraemia and how to screen and identify the prognostic biomarker to predict potential complications in endocarditis patients are urgently required.

Several potential biomarkers such as C-reactive protein (CRP) and erythrocyte sedimentation rate (ESR), have been suggested to use in all patients suspected for IE. However, these biomarkers lack accuracy to differentiate patients with or without IE [Kocazeybek 2003; Cornelissen 2013; Watkin 2007]. Other biomarkers, such as N-terminal-pro-B-type natriuretic peptide (NT-proBNP) alone or in combination with Cystatin C (Cys C), lipopolysaccharide-binding protein (LBP), troponins, aquaporin-9
(AQP9), S100 calcium binding protein A11 (S100A11), E-selectin $(C D 62 E)$, vascular cell adhesion protein 1 (VCAM-1), interleukin-6 (IL-6), rheumatoid factor (RF), and procalcitonin (PCT), have been suggested to detect IE [Snipsoyr 2016], but they have not yet been used clinically to diagnose IE (S1 Table). After reviewing literature and data mining using Open Targets Platform (https://www. targetvalidation.org/), IE associated antigens showing elevated autoantibody responses were established [Koscielny 2017] and are shown in S2 Table. Finding diagnostic biomarkers that could reduce the delayed diagnosis time from IE onset provide the information for early initiation of the correct treatment and have the potential to reduce overall mortality.

Our pilot study on five subjects with more than 1,600 antigens screened on the Sengenics Immunome ${ }^{\mathrm{TM}}$ Protein Array provided a general outlook on autoantibody biomarker profiles of vertebral osteomyelitis subjects [Chen 2020]. The Immunome ${ }^{\mathrm{TM}}$ Protein Array utilizes an immunomics technique using a biotin carboxyl carrier protein $(B C C P)$ domain affinity tag to promote fusion of post-translational biotinylated proteins to an array surface, which includes 1,631 proteins that play important functions in humans, including kinases and transcription factors.

This study has identified protein signature expressed in IE patients with different disease severity through Immunome ${ }^{\mathrm{TM}}$ 


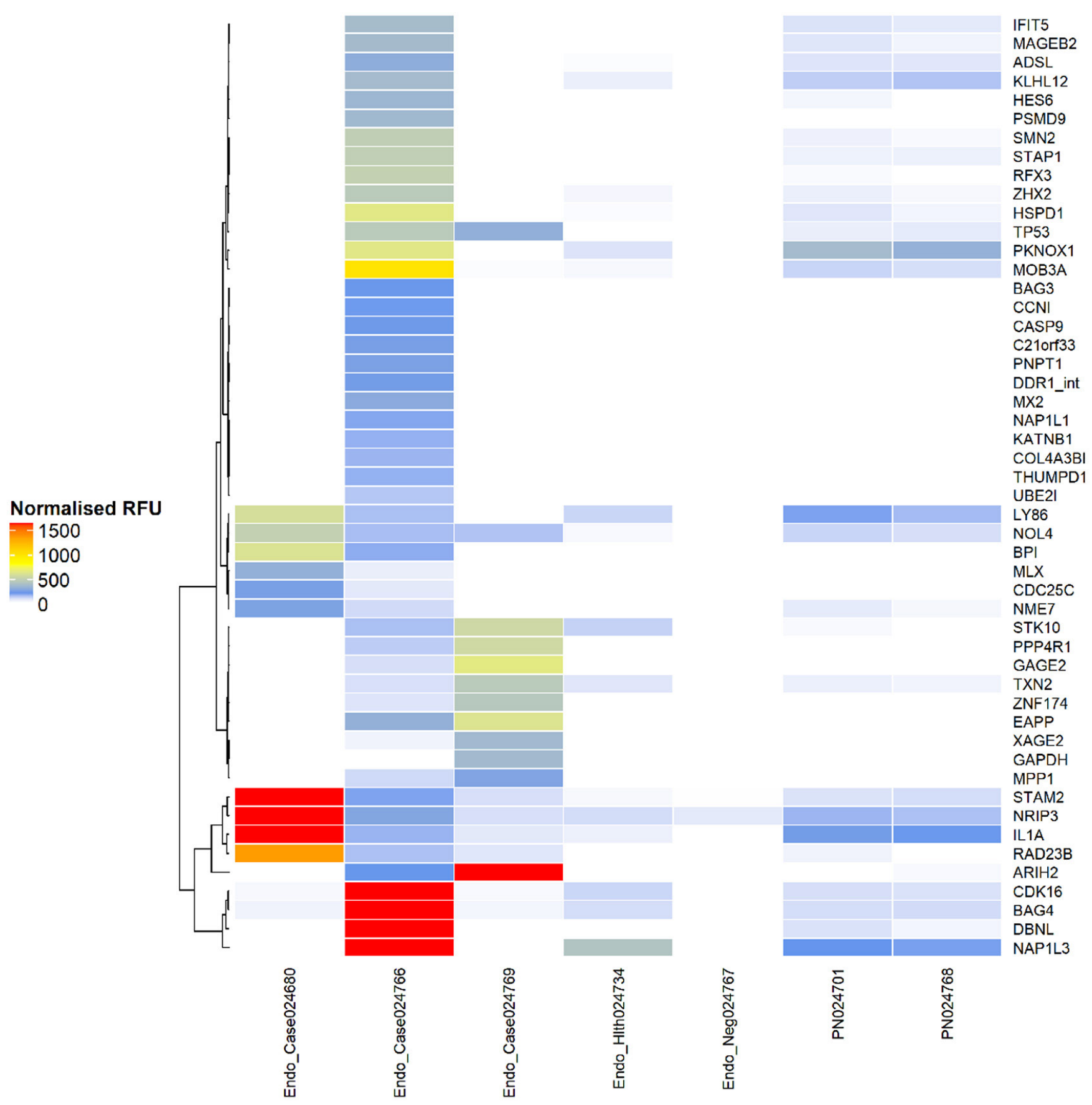

Figure 3. Unsupervised clustering of individual fold changes profiles for 50 biomarkers across 3 IE patient samples, 1 non-IE control, 1 healthy control and 2 pooled normal sera. The clustering was performed based on Ward's method and distance is calculated based on Euclidean distance. RFU, relative fluorescence units. IE: infective endocarditis. Legend: Endo_Case024680, Endo_Case024766 and Endo_Case024769 are 3 IE patient samples ; Endo_Health024734 is 1 healthy control sample; Endo_Neg024767 is 1 non-IE patient sample; PN024701 and PN024768 are 2 pooled normal sera.

profiling. These differential expressed proteins may act as the markers for different IE classifications and further provide insights into potential pathways of IE progression and enable the discovery of novel treatment strategies and appropriate clinical managements for IE patients.

\section{MATERIALS AND METHODS}

Ethics statement: This study was approved by the Ethics Committee of the Changhua Christian Hospital (CCH IRB No. 180905) to allow the collection of blood samples. Each 
Table 1. Summary of clinical data from 5 subjects*

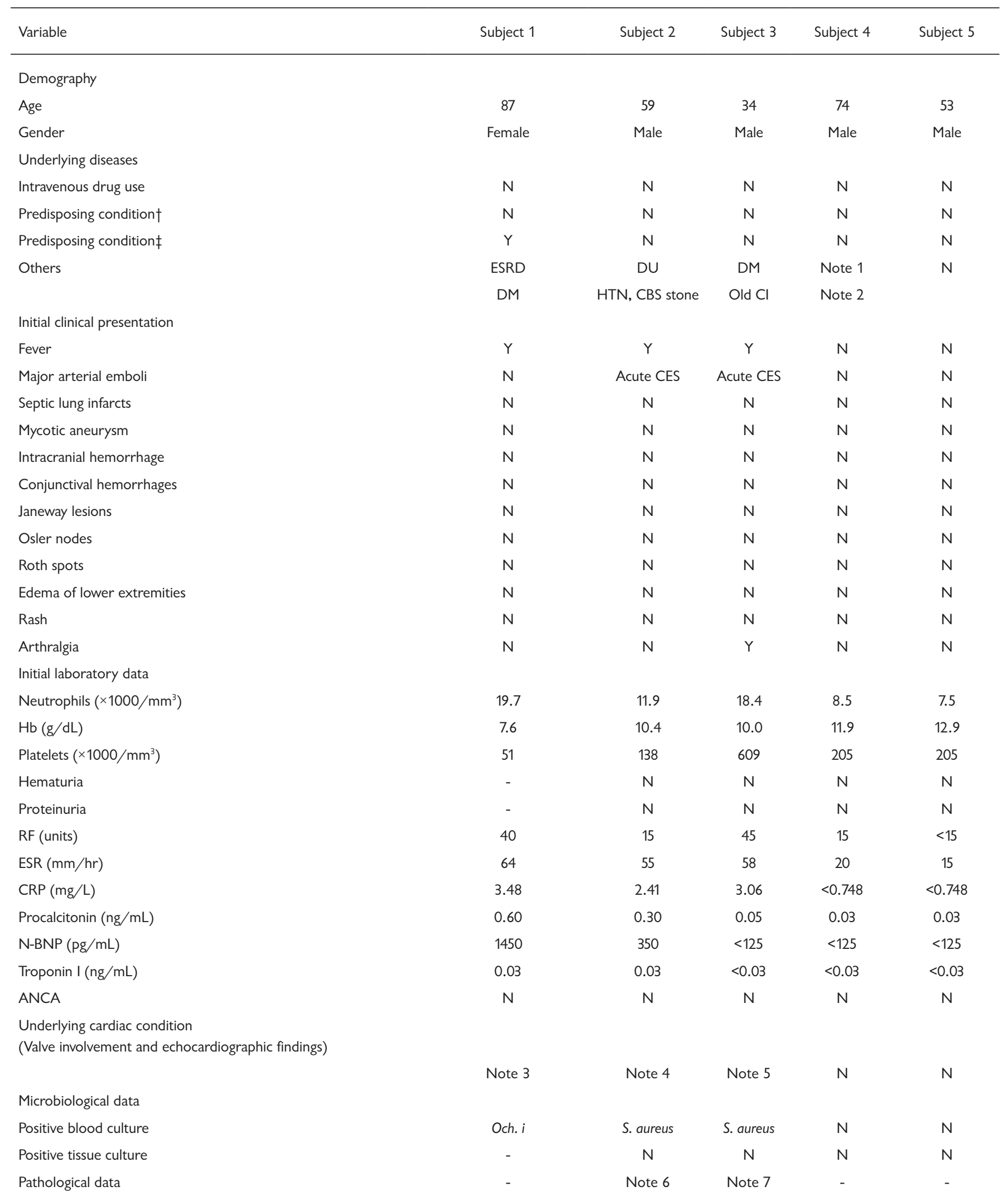


Table 1. Summary of clinical data from 5 subjects [CONTINUED]*

\begin{tabular}{|c|c|c|c|c|c|}
\hline Sampling day & D5 & D4 & D6 & D6 & - \\
\hline Medical treatment (final regimen) & MER+AMK & OXA+AMK & OXA & $\mathrm{N}$ & $\mathrm{N}$ \\
\hline Outcome (until Day 42) & Survive & Survive & Survive & Survive & Survive \\
\hline
\end{tabular}

*Subject one and subject two and subject three are clinical definitive endocarditis cases. Subject four is endocarditis negative-control case. Subject five is healthy control case. $\nmid$ Prosthetic heart valve. $\ddagger A$ valve lesion associated with significant regurgitation or turbulence of blood flow

Abbreviations: acute CES, acute cerebral embolic stroke; ANCA, Antineutrophil cytoplasmic antibodies; AMK, amikacin; CBD stone, common bile duct stones; CRP, C-reactive protein; DM, Type 2 diabetes mellitus; DU, duodenal ulcer; ESR, Erythrocyte sedimentation rate; ESRD, end stage renal diseases; HTN, hypertension; MER, Meropenem; MVR, mitral valve replacement; N-BNP, N-terminal prohormone of brain natriuretic peptide; old Cl, old cerebral infarction. Och. i, Ochrobacturm intermedium; OXA, Oxacillin; RF, rheumatoid factor; S. aureus, Staphylococcus aureus.

Note 1. Coronary artery disease - three vessel diseases. Chronic total occlusion of left anterior descending artery and left circumflex lesion, and right coronary artery ostial to mid right coronary artery diffuse calcified lesion c/w non-ST elevation myocardial infarction, s/p failed percutaneous coronary intervention; post coronary artery bypass graft under partial Extracorporeal Circulation + V-A Extra-Corporeal Membrane Oxygenation set up

Note 2. Coronary artery disease, s/p coronary artery bypass graft + V-A Extra-Corporeal Membrane Oxygenation post hematoma evacuation + remove V-A Extra-Corporeal Membrane Oxygenation + repair right common femoral artery and common femoral vein

Note $3.1 .35 * 0.67 \mathrm{~cm}$ at left coronary cusp of aortic valve

Note 4. A vegetation over anterior leaflet of mitral valve with size $0.989 * 1.92 \mathrm{~cm}$

Note 5. Vegetation $(1.08 * 0.34 \mathrm{~cm})$ over posterior leaflet of mitral valve with mild mitral regurgitation

Note 6. Pathological diagnosis is "Heart, mitral valve, replacement, vegetative endocarditis."

Note 7. Pathological diagnosis is "Mitral valve, robotic assisted MVP with triangular resection, vegetative endocarditis."

Note 8. Coronary artery bypass graft + V-A Extra-Corporeal Membrane Oxygenation post hematoma evacuation + remove V-A Extra-Corporeal Membrane Oxygenation + repair right common femoral artery and common femoral vein

participant provided written informed consent under a protocol that was approved by the institutional review board, and all methods were performed in accordance with these guidelines.

Setting and study design: The population living in the rural areas of Central Taiwan is mostly served by the Changhua Christian Hospital System (CCHS), which consists of 4,000 beds. The Changhua Christian Hospital $(\mathrm{CCH})$ is the largest hospital among nine branch hospitals in the CCHS and is an 1,800-bed tertiary referral medical center situated in Central Taiwan. This study was conducted in the CCHS and approved by the institutional review board of CCH. Subjects of IE, according to modified Duke's criteria [Nishimura 2014], were enrolled after signing written informed consent, and a case-control prospective study was conducted between July 1, 2018 and December 31, 2018, in the CCHS. Each subject had a medical chart that contained medical diagnoses, surgical interventions, and other key information from the medical records. A standardized case report form was used to collect data on age, sex, site of involvement, underlying disease, clinical presentation, length of symptoms, diagnostic laboratory and radiographic reports, medical treatment, interventions, and outcomes. Concomitant diseases included hypertension, chronic kidney disease, diabetes, hyperlipidemia, preexisting cardiovascular disease, and previous stroke. Stroke, heart failure, atrial fibrillation, and congestive heart failure were identified by taking a history and by medical chart review. Mortality attributable to IE was defined death within six weeks of effective antimicrobial agents. A follow-up record was from the date of diagnosis to the date of death or to the date of the last available record.

Microbiological evaluation, serological examinations, and echocardiographic examinations: Blood samples routinely were sent for tests for complete whole blood cell counts. Blood was cultured in the BD BACTEC FX (Becton Dickinson, Sparks, MD, USA) culture system and identified by using matrix-assisted laser desorption ionization-time of flight mass spectrometry (bioMerieux, Hazelwood, MO, USA). The serologic examinations were performed as follows: rheumatoid factor using enzyme-linked immunosorbent assay (normal range, $\leq 6.0$ Units), C-reactive protein using near infrared particle immunoassay rate methodology (normal range, $<0.748 \mathrm{mg} / \mathrm{dL}$ ), procalcitonin using electrochemiluminescence immunoassay (normal range, $<0.5 \mathrm{ng} /$ $\mathrm{mL}$ ), N-terminal prohormone of brain natriuretic peptide using electrochemiluminescence immunoassay (normal range, $<0.125 \mathrm{pg} / \mathrm{mL}$ ), Troponin I using two-site immunoenzymatic assay (normal range, $<0.03 \mathrm{ng} / \mathrm{mL}$ ), and antineutrophil cytoplasmic antibodies using fluorescence enzyme immunoassay (normal range, $<2.0 \mathrm{IU} / \mathrm{mL}$ ). Authors used a modified Simpson's method to calculate $\mathrm{LV}$ ejection fraction as described by the American Echocardiographic Society [Lang 2005].

Definitions for IE: Authors included only patients who met modified Duke criteria for definite or possible IE [Nishimura 2014]. Definite IE defines patients with pathological criteria (microorganisms demonstrated by culture or histologic 
Table 2. List of top 10 shortlisted antigens from the Immunome ${ }^{T M}$ protein microarray platform with significant autoantibody responses in infective endocarditis patients' samples versus non-IE control

\begin{tabular}{|c|c|c|c|c|c|c|c|}
\hline 2 & RAD23B & $\begin{array}{l}\text { UV excision repair protein } \\
\text { RAD23 homolog B }\end{array}$ & P54727 & 3 & 100 & 7.333 & 77.709 \\
\hline 5 & $A R I H 2$ & $\begin{array}{l}\text { E3 ubiquitin-protein ligase } \\
\text { ARIH2 }\end{array}$ & O95376 & 2 & 66.667 & 24.656 & 74.44 \\
\hline 6 & TP53 & Cellular tumor antigen p53 & P04637 & 2 & 66.667 & 6.561 & 68.656 \\
\hline 10 & XAGE2 & $X$ antigen family member 2 & Q96GT9 & 2 & 66.667 & 5.479 & 55.327 \\
\hline
\end{tabular}

IE, infective endocarditis

examination or a histologic examination showing active endocarditis); or patients with two major criteria (positive blood culture and evidence of endocardial involvement by echocardiogram), one major criterion and three minor criteria (minor criteria: predisposing heart conditions or intravenous drug use, fever, vascular phenomena, immunologic phenomena, and microbiologic evidence that do not meet the major criteria) or five minor criteria [Nishimura 2014]. Possible IE defines patients with one major and one minor criterion or three minor criteria [Nishimura 2014].

Quality report: The Sengenics Immunome ${ }^{\mathrm{TM}}$ Protein Array platform was used for high-throughput quantification of autoantibodies in five sera samples collected from IE subjects, one non-IE control (cardiac-disease patient without infection), and one healthy control, which were analyzed together with two pooled normal sera (Sengenics Internal quality control samples). All samples passed quality control parameters that evaluated quantitative metrics related to the array and assay quality and consistency of results. The results showed exceptional data consistency, which is measured as a percentage of the coefficient of variance $(\mathrm{CV} \%)$. The intraprotein, intra-slide, and inter-array $\mathrm{CV} \%$ for all proteins and control probes was below the quality control limit of $15 \%$.

Bioinformatics methodology: The Immunome ${ }^{\mathrm{TM}}$ Protein Array protocol is shown on the Sengenics website (http://www.sengenics.com/wp-content/ uploads/2019/07/Sengenics-KREX-User-ManualJune-2019.pdf). In brief, the penetrance-based fold change analysis method was conducted between IE patients and negative and healthy controls to identify highly expressed proteins in each case sample. A step-by-step description of this method is as follows:

Step 1: Individual fold changes for both case and control were calculated by dividing the value of relative fluorescence units (RFU) for each protein in each sample, $\mathrm{H}$, by the mean of the RFU values of each protein across all control samples (i.e., background threshold).

Equation 1: Individual fold change $(\mathrm{FC})=$ HCase or Controlp (HControl)

Step 2: For proteins with individual fold change of less than 2-fold above the background threshold, their signal intensities RFU were replaced with zeros.

Step 3: Penetrance frequency (number of case and control samples with individual fold changes $\geq 2$-fold) for both case (frequency case) groups was determined for each protein.

Equation 2: Frequency Case $=n$ (Individual FC $($ Case $)) \geq 2$ )

Equation 3: Frequency Control=n (Individual FC (Control)) $\geq 2$ )

Step 4: Penetrance fold changes for both case and control groups were calculated for each protein.

Equation 4: Penetrance fold Changecase $=\mu($ HControl[i] $) /$ $\mu$ (HControl)

$[i]=$ HCase with FC Case $\geq 2$-fold

An unsupervised clustering was performed for the following biomarkers across patients, controls, and pooled normal samples using a hierarchical clustering method, i.e. Ward's method [Ward 1965] and distance calculated based on Euclidean distance. The heatmap shown in Figures 2-3 were plotted using ComplexHeatmap v 1.20.0 package [Gu 2016] in Bioconductor (Figures 2 and 3). 
Table 3. List of top 10 shortlisted antigens from the Immunome ${ }^{T M}$ protein microarray platform with significant autoantibody responses in endocarditis patients' samples versus healthy control

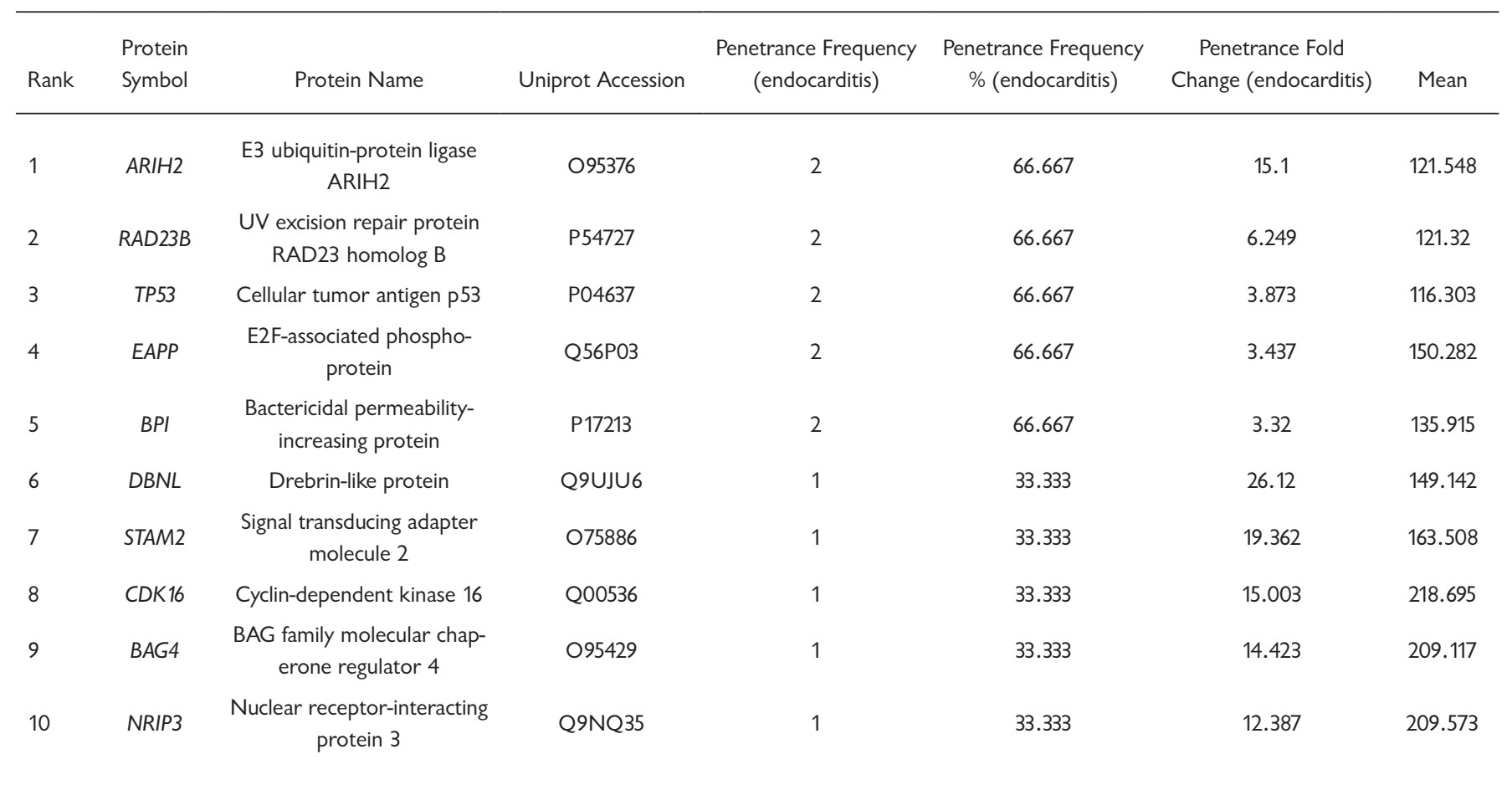

\section{RESULTS}

Subject characteristics: Five subjects, including three IE subjects, one non-IE control subject (cardiac-disease patient without infection), and one healthy control subject, were enrolled during the period from July 1, 2018 to December 31, 2018. The study protocol is described in Figure 1. Of the five subjects, two had diabetes mellitus, two were hypertensive, and one had end-stage renal disease. None of the subjects had intravenous drug use or a predisposing heart condition (prosthetic heart valve or a valve lesion associated with significant regurgitation or turbulence of blood flow). For the initial signs and symptoms before admission, three had fever. However, none of the following symptoms were found: major arterial emboli, septic pulmonary infarcts, mycotic aneurysm, intracranial haemorrhage, conjunctival haemorrhages, Janeway lesions, Osler nodes, and Roth spots. For the initial laboratory data, three had leucocytosis, elevated ESR and CRP; two had elevated RF and PCT; and one had elevated NT-proBNP. Initial blood culture showed the presence of Staphylococcus aureus in two subjects and Ochrobacturm intermedium in one subject. The following cardiac echogram showed vegetation over the mitral valve in two subjects and vegetation over the aortic valve in one subject. Overall, all of three subjects survived. Reported potential biomarkers for IE, including ESR, CRP, RF, PCT, and NT-proBNP, were found to be partially significant. In our study, we found the number of neutrophils, as well as the ESR and CRP, in IE patients were significantly higher than non-IE control and healthy control. In contrast, the concentration of hemoglobin in IE patients was lower than non-IE control and healthy control (Table 1).
Biomarker identification using penetrance fold change analysis: The penetrance fold change-based method was performed for the following two studies. First, analysis of IE sera versus non-IE control sera was conducted; Table 2 shows the top 10 antigens with high autoantibody titres identified by Sengenics Immunome ${ }^{\mathrm{TM}}$ Protein Array. Penetrance fold changes between IE sera and non-IE control were as follows: E3 ubiquitin-protein ligase ARIH2 (ARIH2), 24.656; Interleukin-1 alpha (IL1A), 8.503; UV excision repair protein RAD23 homolog B (RAD23B), 7.333; Cellular tumour antigen p53 (TP53), 6.561; E2F-associated phosphoprotein $(E A P P), 5.97 ; \mathrm{G}$ antigen 2B/2C (GAGE2), 5.669; Glyceraldehyde-3-phosphate dehydrogenase $(G A P D H), 5.667 ; \mathrm{X}$ antigen family member 2 (XAGE2), 5.479; Nucleolar protein 4 (NOL4), 3.195; and Tudor and KH domain-containing protein $(T D R K H), 2.408$.

Second, analysis of IE sera versus healthy control (healthy person) sera was performed; Table 3 shows the top 10 antigens with high autoantibody titres identified from the analysis. Penetrance fold changes between IE sera and the healthy control were as follows: Drebrin-like protein $(D B N L), 26.12$; Signal transducing adapter molecule 2 (STAM2), 19.362; ARIH2, 15.1; Cyclin-dependent kinase 16 (CDK16), 15.003; BAG family molecular chaperone regulator 4 (BAG4), 14.423; Nuclear receptor-interacting protein 3 (NRIP3), 12.387; RAD23B, 6.249; TP53, 3.873; EAPP, 3.437; and Bactericidal permeability-increasing protein $(B P I), 3.32$. Taken together, we found the penetrance fold changes of $A R I H 2, R A D 23 B$, TP53, and EAPP were in the top list of both the IE versus non-IE control and IE versus healthy control. 
Table S1. Summary of biomarkers identified from literature

\begin{tabular}{l} 
Year/Country/Author \\
\hline 2017/France/Zaratzian C \\
2017/Guangdong, China/Wei XB \\
2016/Japan/Hirai K \\
2016/India/Thoker ZA \\
2016/CHU Rouen, France/Langlois V \\
2016/Aarhus, Denmark/Snipsøyr MG \\
2015/Taiwan/Yeh CY \\
2015/Netherlands/Schoffelen T \\
2014/Bad Oeynhausen, Germany/Weinstock M \\
2014/Marseille, France/Ka MB \\
2014/Shanghai, China/Ying CM \\
2014/Valladolid, Spain/Bustamante J \\
2013/Taiwan/Yu CW \\
2013/Tours, France/Lanotte P \\
2014/Osaka, Japan/Okada Y \\
2011/Japan/Satake K \\
2007/FL, USA/Chirinos JA \\
2003/Turkey/Kocazeybek B \\
200therlands/Verhagen DW \\
2015asa H
\end{tabular}

Biomarker

Reference

DOI: $10.1007 / s 10096-017-2903-1$

DOI: $10.1177 / 2047487316686436$

DOI: 10.2169/internalmedicine.55.7331

DOI: 10.1016/j.ijcard.2016.07.130

DOI: $10.1097 / M D .0000000000002564$

DOI: 10.1016/j.jjcard.2015.09.028

Cystatin C, lipopolysaccharide-binding protein, troponins, aquaporin-9, S100 calcium binding protein

A11, E-selectin, VCAM-1, interleukin-6 (IL-6)

interleukin-17

interferon-gamma IL-2

interleukin-1

IL-10 in PD-1 modulation

antineutrophil cytoplasmic antibody

IL-6 IL-8 IFN-

procalcitonin

exodeoxyribonuclease VII 50S ribosomal protein

L7/L12

CsbD-like protein Thioredoxin

CRP levels

PR3-ANCA

PR3-ANCA

Antineutrophil cytoplasmic antibodies

C-reactive protein

Procalcitonin C-reactive protein
DOI: 10.1128/IAI.02965-14

DOI: $10.3389 /$ fmicb.2015.00093

DOI: 10.1371/journal.pone.0110151

DOI: 10.1371/journal.pone.0107533

DOI: 10.1371/journal.pone.0089777

DOI: $10.1111 /$ apm. 12189

DOI: 10.1016/j.ajem.2013.03.008

DOI: 10.1371/journal.pone.0054393

PMID: 23411851

DOI: $10.1007 /$ s10165-011-0434-7

PMID: 22989831

DOI: $10.1007 / s 10067-005-0176-z$

DOI: $10.1007 / \mathrm{s} 10096-007-0319-z$

DOI: $10.1159 / 000069777$
For the further analysis, the penetrance percentage frequency of IL1A, NOL4, and TDRKH between IE patients and the non-IE control were $100 \%$, and the penetrance fold change were 8.503, 3.195 and 2.408, respectively (Table 2). The penetrance percentage frequency of GAGE2, GAPDH and $X A G E 2$ between IE patients and the non-IE control were $66.667 \%$, and the penetrance fold change were 5.669, 5.667 and 5.479 , respectively (Table 2). Moreover, the penetrance percentage frequency of BPI between IE patients and the healthy control was $66.667 \%$, and the penetrance fold change was 3.32 (Table 3). The penetrance percentage frequency of DBNL, STAM2, CDK16, BAG4, and NRIP3 between IE patients and the healthy control were $33.333 \%$, and the penetrance fold change were $26.12,19.362,15.003,14.423$ and 12.387 , respectively (Table 3 ).

Auto-antibody profile across all cases, controls, and pooled normal samples against the top 10 antigens for IE: Comparison of autoantibody profiles among IE, non-IE control, healthy control, and pooled normal samples for the most clinically significant common biomarkers identified based on penetrance fold change analysis were shown in Figure 2. All antigens were elevated in the three IE samples. Individually, $B P I, I L 1 A$, and RAD23B were elevated in subject $1 ; A R I H 2$, $B P I, E A P P, G A G E 2, R A D 23$ and TP53 were elevated in subject 2; and ARIH2, EAPP, GAGE2, GAPDH, TP53, XAGE2, and Zinc finger protein 174 (ZNF174) were elevated in subject 3 . Since the outcome of subject 1 is the worst one of all patients, the high expressions of BPI, IL1A, and RAD23B may have the potential to be the prognosis markers of IE patients.

Auto-antibody profile of relevant biomarker hits: Figure 3 shows the top 50 common biomarkers across the IE samples, non-IE control, healthy control, and pooled normal sera based on the penetrance fold change. Clustering was performed based on Ward's method, and the Euclidean distance was calculated. Unsupervised clustering of individual fold change profiles for 50 biomarkers among IE, non-IE control, healthy control, and pooled normal samples is shown in Figure 3. All antigens were elevated among the three IE 
Table S2. Proposed functional annotation biomarkers for heart disease from Open Targets platform

No. of associ-
ated targets $\quad$ All targets

Endocarditis

IL1A (Interleukin-1 alpha)

GAPDH(Glyceraldehyde3-phosphate dehydrogenase)
Rheumatic Heart Disease
3
HSPD1 (heat shock protein family D member 1, Hsp60)
Receptor for IL1A, IL1B and IL1RN. After binding to interleukin-1 associates with the coreceptor IL1RAP to form the high affinity interleukin-1 receptor complex which mediates interleukin-1-dependent activation of NF-kappa-B, MAPK and other pathways. Signaling involves the recruitment of adapter molecules such as TOLLIP, MYD88, and IRAK1 or IRAK2 via the respective TIR domains of the receptor/coreceptor subunits. Binds ligands with comparable affinity and binding of antagonist IL1RN prevents association with IL1RAP to form a signaling complex. Involved in IL1B-mediated costimulation of IFNG production from T-helper 1 (Th1) cells

Has both glyceraldehyde-3-phosphate dehydrogenase and nitrosylase activities, thereby playing a role in glycolysis and nuclear functions, respectively. Participates in nuclear events including transcription, RNA transport, DNA replication and apoptosis. Nuclear functions are probably due to the nitrosylase activity that mediates cysteine S-nitrosylation of nuclear target proteins such as SIRT1, HDAC2 and PRKDC. Modulates the organization and assembly of the cytoskeleton. Facilitates the CHP1-dependent microtubule and membrane associations through its ability to stimulate the binding of CHP1 to microtubules (By similarity). Glyceraldehyde-3-phosphate dehydrogenase is a key enzyme in glycolysis that catalyzes the first step of the pathway by converting D-glyceraldehyde 3-phosphate (G3P) into 3-phospho-D-glyceroyl phosphate. Component of the GAIT (gamma interferon-activated inhibitor of translation) complex which mediates interferongamma-induced transcript-selective translation inhibition in inflammation processes. Upon interferon-gamma treatment assembles into the GAIT complex which binds to stem loop-containing GAIT elements in the 3'-UTR of diverse inflammatory mRNAs (such as ceruplasmin) and suppresses their translation

Chaperonin implicated in mitochondrial protein import and macromolecular assembly. Together with $\mathrm{Hsp} 10$, facilitates the correct folding of imported proteins. May also prevent misfolding and promote the refolding and proper assembly of unfolded polypeptides generated under stress conditions in the mitochondrial matrix (PubMed:1346131, PubMed:11422376). The functional units of these chaperonins consist of heptameric rings of the large subunit $\mathrm{Hsp} 60$, which function as a back-to-back double ring. In a cyclic reaction, Hsp60 ring complexes bind one unfolded substrate protein per ring, followed by the binding of ATP and association with 2 heptameric rings of the co-chaperonin Hsp10. This leads to sequestration of the substrate protein in the inner cavity of Hsp60 where, for a certain period of time, it can fold undisturbed by other cell components. Synchronous hydrolysis of ATP in all Hsp60 subunits results in the dissociation of the chaperonin rings and the release of ADP and the folded substrate protein (Probable).

Receptor for IL1A, IL1B and IL1RN. After binding to interleukin-1 associates with the coreceptor IL1RAP to form the high affinity interleukin-1 receptor complex which mediates interleukin-1-dependent activation of NF-kappa-B, MAPK and other pathways. Signaling involves the recruitment of adapter molecules such as TOLLIP, MYD88, and IRAK1 or

IL1A (Interleukin-1 alpha)

IRAK2 via the respective TIR domains of the receptor/coreceptor subunits. Binds ligands with comparable affinity and binding of antagonist IL1RN prevents association with IL1RAP to form a signaling complex. Involved in IL1B-mediated costimulation of IFNG production from T-helper 1 (Th1) cells

PKNOX1(PBX/knotted 1 homeobox 1)
Activates transcription in the presence of PBX1A and HOXA1 
Table S2. Proposed functional annotation biomarkers for heart disease from Open Targets platform [CONTINUED]

\begin{tabular}{lcc}
\hline Disease full name & $\begin{array}{c}\text { No. of associ- } \\
\text { ated targets }\end{array}$ & All targets \\
\hline Heart disease & 22 & BAG3 (BCL2 associated \\
athanogene 3)
\end{tabular}

TP53 (Cellular tumor antigen $\mathrm{p} 53$ )

PPP4R1 (protein phosphatase 4 regulatory subunit 1 )

RFX3 (regulatory factor X3)

SMN2 (survival of motor neuron 2, centromeric)
Co-chaperone for HSP70 and HSC70 chaperone proteins. Acts as a nucleotide-exchange factor (NEF) promoting the release of ADP from the HSP70 and HSC70 proteins thereby triggering client/substrate protein release. Nucleotide release is mediated via its binding to the nucleotide-binding domain (NBD) of HSPA8/HSC70 where as the substrate release is mediated via its binding to the substrate-binding domain (SBD) of HSPA8/HSC70 (PubMed:9873016, PubMed:27474739). Has anti-apoptotic activity (PubMed:10597216). Plays a role in the HSF1 nucleocytoplasmic transport (PubMed:26159920).

lipid binding

Component of the EKC/KEOPS complex that is required for the formation of a threonylcarbamoyl group on adenosine at position 37 (t6A37) in tRNAs that read codons beginning with adenine (PubMed:22912744, PubMed:27903914). The complex is probably involved in the transfer of the threonylcarbamoyl moiety of threonylcarbamoyl-AMP

(TC-AMP) to the N6 group of A37 (PubMed:22912744, PubMed:27903914). TP53RK has ATPase activity in the context of the EKC/KEOPS complex and likely plays a supporting role to the catalytic subunit OSGEP (By similarity). Atypical protein kinase that phosphorylates 'Ser-15' of $\mathrm{p} 53 / \mathrm{TP} 53$ protein and may therefore participate in its activation (PubMed:11546806)

Regulatory subunit of serine/threonine-protein phosphatase 4. May play a role in regulation of cell division in renal glomeruli. The PPP4C-PPP4R1 PP4 complex may play a role in dephosphorylation and regulation of $\mathrm{HDAC} 3$

Transcription factor required for ciliogenesis and islet cell differentiation during endocrine pancreas development. Essential for the differentiation of nodal monocilia and left-right asymmetry specification during embryogenesis. Required for the biogenesis of motile cilia by governing growth and beating efficiency of motile cells. Also required for ciliated ependymal cell differentiation. Regulates the expression of genes involved in ciliary assembly (DYNC2LI1, FOXJ1 and BBS4) and genes involved in ciliary motility (DNAH11, DNAH9 and DNAH5) (By similarity). Together with RFX6, participates in the differentiation of 4 of the 5 islet cell types during endocrine pancreas development, with the exception of pancreatic PP (polypeptide-producing) cells. Regulates transcription by forming a heterodimer with another RFX protein and binding to the X-box in the promoter of target genes (PubMed:20148032). Represses transcription of MAP1A in non-neuronal cells but not in neuronal cells (PubMed:12411430).

The SMN complex plays a catalyst role in the assembly of small nuclear ribonucleoproteins (snRNPs), the building blocks of the spliceosome. Thereby, plays an important role in the splicing of cellular pre-mRNAs. Most spliceosomal snRNPs contain a common set of Sm proteins SNRPB, SNRPD1, SNRPD2, SNRPD3, SNRPE, SNRPF and SNRPG that assemble in a heptameric protein ring on the $\mathrm{Sm}$ site of the small nuclear RNA to form the core snRNP. In the cytosol, the Sm proteins SNRPD1, SNRPD2, SNRPE, SNRPF and SNRPG are trapped in an inactive $6 \mathrm{~S}$ pICln-Sm complex by the chaperone CLNS1A that controls the assembly of the core snRNP. Dissociation by the SMN complex of CLNS1A from the trapped Sm proteins and their transfer to an SMN-Sm complex triggers the assembly of core snRNPs and their transport to the nucleus. Ensures the correct splicing of $U 12$ intron-containing genes that may be important for normal motor and proprioceptive neurons development. Also required for resolving RNA-DNA hybrids created by RNA polymerase II, that form R-loop in transcription terminal regions, an important step in proper transcription termination. May also play a role in the metabolism of small nucleolar ribonucleoprotein (snoRNPs). 
Table S2. Proposed functional annotation biomarkers for heart disease from Open Targets platform [CONTINUED]

Disease full name $\quad \begin{gathered}\text { No. of associ- } \\ \text { ated targets } \quad \text { All targets }\end{gathered}$

ARIH2 (ariadne RBR E3 ubiquitin protein ligase 2)

CASP9 (caspase 9)

NME7 (NME/NM23 family member 7)

MLX (MAX dimerization protein $M L X)$

HSPD1 (heat shock protein family D (Hsp60) member 1)

PKNOX1 (PBX/knotted 1 homeobox 1)

ADSL (adenylosuccinate lyase)
E3 ubiquitin-protein ligase, which catalyzes ubiquitination of target proteins together with ubiquitin-conjugating enzyme E2 UBE2L3 (PubMed:16118314, PubMed:17646546, PubMed:19340006, PubMed:24076655). Acts as an atypical E3 ubiquitin-protein ligase by working together with cullin-5-RING ubiquitin ligase complex (ECS complex, also named CRL5 complex) and initiating ubiquitination of ECS substrates: associates with ECS complex and specifically mediates addition of the first ubiquitin on ECS targets

(By similarity). The initial ubiquitin is then elongated (By similarity). E3 ubiquitinprotein ligase activity is activated upon binding to neddylated form of the ECS complex

(PubMed:24076655). Mediates 'Lys-6', 'Lys-48'-and 'Lys-63'-linked polyubiquitination (PubMed:16118314, PubMed:17646546, PubMed:19340006). May play a role in myelopoiesis (PubMed:19340006)

Involved in the activation cascade of caspases responsible for apoptosis execution. Binding of caspase- 9 to Apaf- 1 leads to activation of the protease which then cleaves and activates caspase-3. Promotes DNA damage-induced apoptosis in a ABL1/c-Abl-dependent manner. Proteolytically cleaves poly(ADP-ribose) polymerase (PARP). Isoform 2 lacks activity is an dominant-negative inhibitor of caspase-9.

Major role in the synthesis of nucleoside triphosphates other than ATP. The ATP gamma phosphate is transferred to the NDP beta phosphate via a ping-pong mechanism, using a phosphorylated active-site intermediate.

Transcription regulator. Forms a sequence-specific DNA-binding protein complex with MAD1, MAD4, MNT, WBSCR 14 and MLXIP which recognizes the core sequence 5'-CACGTG-3'. The TCFL4-MAD1, TCFL4-MAD4, TCFL4-WBSCR14 complexes are transcriptional repressors. Plays a role in transcriptional activation of glycolytic target genes. Involved in glucose-responsive gene regulation.

Chaperonin implicated in mitochondrial protein import and macromolecular assembly.

Together with Hsp10, facilitates the correct folding of imported proteins. May also prevent misfolding and promote the refolding and proper assembly of unfolded polypeptides generated under stress conditions in the mitochondrial matrix (PubMed:1346131, PubMed:11422376). The functional units of these chaperonins consist of heptameric rings of the large subunit $\mathrm{Hsp} 60$, which function as a back-to-back double ring. In a cyclic reaction, Hsp60 ring complexes bind one unfolded substrate protein per ring, followed by the binding of ATP and association with 2 heptameric rings of the co-chaperonin Hsp10. This leads to sequestration of the substrate protein in the inner cavity of Hsp60 where, for a certain period of time, it can fold undisturbed by other cell components. Synchronous hydrolysis of ATP in all Hsp60 subunits results in the dissociation of the chaperonin rings and the release of ADP and the folded substrate protein (Probable).

DNA-binding transcription activator activity, RNA polymerase II-specific DNA-binding transcription factor activity DNA-binding transcription factor activity, RNA polymerase II-specific protein heterodimerization activity sequence-specific DNA binding

Catalyzes two non-sequential steps in de novo AMP synthesis: converts (S)-2-(5-amino1-(5-phospho-D-ribosyl)imidazole-4-carboxamido)succinate (SAICAR) to fumarate plus 5-amino-1-(5-phospho-D-ribosyl)imidazole-4-carboxamide, and thereby also contributes to de novo IMP synthesis, and converts succinyladenosine monophosphate (SAMP) to AMP and fumarate 
Table S2. Proposed functional annotation biomarkers for heart disease from Open Targets platform [CONTINUED]

No. of associ-
ated targets $\quad$ All targets

KATNB1 (katanin regulatory subunit B1)

RAD23B (RAD23 homolog

$B$, nucleotide excision repair protein)

DDR1 (discoidin domain receptor tyrosine kinase 1)
Participates in a complex which severs microtubules in an ATP-dependent manner. May act to target the enzymatic subunit of this complex to sites of action such as the centrosome. Microtubule severing may promote rapid reorganization of cellular microtubule arrays and the release of microtubules from the centrosome following nucleation. Microtubule release from the mitotic spindle poles may allow depolymerization of the microtubule end proximal to the spindle pole, leading to poleward microtubule flux and poleward motion of chromosome. Microtubule release within the cell body of neurons may be required for their transport into neuronal processes by microtubule-dependent motor proteins. This transport is required for axonal growth.

Multiubiquitin chain receptor involved in modulation of proteasomal degradation. Binds to polyubiquitin chains. Proposed to be capable to bind simultaneously to the $26 \mathrm{~S}$ proteasome and to polyubiquitinated substrates and to deliver ubiquitinated proteins to the proteasome. May play a role in endoplasmic reticulum-associated degradation (ERAD) of misfolded glycoproteins by association with PNGase and delivering deglycosylated proteins to the proteasome.

Involved in global genome nucleotide excision repair (GG-NER) by acting as component of the XPC complex. Cooperatively with CETN2 appears to stabilize XPC. May protect XPC from proteasomal degradation.

The XPC complex is proposed to represent the first factor bound at the sites of DNA damage and together with other core recognition factors, XPA, RPA and the TFIIH complex, is part of the pre-incision (or initial recognition) complex. The XPC complex recognizes a wide spectrum of damaged DNA characterized by distortions of the DNA helix such as single-stranded loops, mismatched bubbles or single-stranded overhangs. The orientation of XPC complex binding appears to be crucial for inducing a productive NER. XPC complex is proposed to recognize and to interact with unpaired bases on the undamaged DNA strand which is followed by recruitment of the TFIIH complex and subsequent scanning for lesions in the opposite strand in a 5'-to-3' direction by the NER machinery. Cyclobutane pyrimidine dimers (CPDs) which are formed upon UV-induced DNA damage esacpe detection by the XPC complex due to a low degree of structural perurbation. Instead they are detected by the UV-DDB complex which in turn recruits and cooperates with the XPC complex in the respective DNA repair. In vitro, the XPC:RAD23B dimer is sufficient to initiate NER; it preferentially binds to cisplatin and UV-damaged double-stranded DNA and also binds to a variety of chemically and structurally diverse DNA adducts. XPC:RAD23B contacts DNA both 5' and 3 ' of a cisplatin lesion with a preference for the $5^{\prime}$ side. XPC:RAD23B induces a bend in DNA upon binding. XPC:RAD23B stimulates the activity of DNA glycosylases TDG and SMUG1.

Tyrosine kinase that functions as cell surface receptor for fibrillar collagen and regulates cell attachment to the extracellular matrix, remodeling of the extracellular matrix, cell migration, differentiation, survival and cell proliferation. Collagen binding triggers a signaling pathway that involves SRC and leads to the activation of MAP kinases. Regulates remodeling of the extracellular matrix by up-regulation of the matrix metalloproteinases MMP2, MMP7 and MMP9, and thereby facilitates cell migration and wound healing. Required for normal blastocyst implantation during pregnancy, for normal mammary gland differentiation and normal lactation. Required for normal ear morphology and normal hearing (By similarity). Promotes smooth muscle cell migration, and thereby contributes to arterial

wound healing. Also plays a role in tumor cell invasion. Phosphorylates PTPN11. 
Table S2. Proposed functional annotation biomarkers for heart disease from Open Targets platform [CONTINUED]

\begin{tabular}{ll}
\hline No. of associ- \\
ated targets
\end{tabular}$\quad$ All targets

IL1A (Interleukin-1 alpha)

GAPDH

TXN2 (thioredoxin 2)

LY86 (lymphocyte antigen

86)

UBE2I (ubiquitin conjugating enzyme E2 I)

PSMD9 (proteasome 26S subunit, non-ATPase 9)
Receptor for IL1A, IL1B and IL1RN. After binding to interleukin-1 associates with the coreceptor IL1RAP to form the high affinity interleukin-1 receptor complex which mediates interleukin-1-dependent activation of NF-kappa-B, MAPK and other pathways. Signaling involves the recruitment of adapter molecules such as TOLLIP, MYD88, and IRAK1 or IRAK2 via the respective TIR domains of the receptor/coreceptor subunits. Binds ligands with comparable affinity and binding of antagonist IL1RN prevents association with IL1RAP to form a signaling complex. Involved in IL1B-mediated costimulation of IFNG production from T-helper 1 (Th1) cells.

Has both glyceraldehyde-3-phosphate dehydrogenase and nitrosylase activities, thereby playing a role in glycolysis and nuclear functions, respectively. Participates in nuclear events including transcription, RNA transport, DNA replication and apoptosis. Nuclear functions are probably due to the nitrosylase activity that mediates cysteine S-nitrosylation of nuclear target proteins such as SIRT1, HDAC2 and PRKDC. Modulates the organization and assembly of the cytoskeleton. Facilitates the CHP1-dependent microtubule and membrane associations through its ability to stimulate the binding of CHP1 to microtubules (By similarity). Glyceraldehyde-3-phosphate dehydrogenase is a key enzyme in glycolysis that catalyzes the first step of the pathway by converting D-glyceraldehyde 3-phosphate (G3P) into 3-phospho-D-glyceroyl phosphate. Component of the GAIT (gamma interferon-activated inhibitor of translation) complex which mediates interferongamma-induced transcript-selective translation inhibition in inflammation processes. Upon interferon-gamma treatment assembles into the GAIT complex which binds to stem loop-containing GAIT elements in the 3'-UTR of diverse inflammatory mRNAs (such as ceruplasmin) and suppresses their translation

Important for the control of mitochondrial reactive oxygen species homeostasis, apoptosis regulation and cell viability. Possesses a dithiol-reducing activity.

May cooperate with CD180 and TLR4 to mediate the innate immune response to bacterial lipopolysaccharide (LPS) and cytokine production. Important for efficient CD180 cell surface expression (By similarity).

Accepts the ubiquitin-like proteins SUMO1, SUMO2, SUMO3, SUMO4 and SUMO1P1/ SUMO5 from the UBLE1A-UBLE1B E1 complex and catalyzes their covalent attachment to other proteins with the help of an E3 ligase such as RANBP2, CBX4 and ZNF451. Can catalyze the formation of poly-SUMO chains. Necessary for sumoylation of FOXL2 and KAT5. Essential for nuclear architecture and chromosome segregation. Sumoylates p53/ TP53 at 'Lys-386'.

Acts as a chaperone during the assembly of the $26 \mathrm{~S}$ proteasome, specifically of the base subcomplex of the PA700/19S regulatory complex (RC). During the base subcomplex assembly is part of an intermediate PSMD9:PSMC6:PSMC3 module, also known as modulator trimer complex; PSMD9 is released during the further base assembly process. samples. Individually, STAM2, NRIP3, IL1A, and RAD23B showed high normalised RFU in subject one; CDK16, BAG4, $D B N L$, and NRIP3 had high RFU in subject two; and ARIH2 had high RFU in subject three.

\section{DISCUSSION}

This study identified biomarkers for IE using the Sengenics Immunome ${ }^{\mathrm{TM}}$ Protein Array platform. We identified several distinct and novel biomarkers in this study conducted in Taiwan.
There were several major findings. First, there was partial significance found for known potential biomarkers of IE, including ESR, CRP, RF, PCT, and NT-proBNP. Second, we observed target antigens with high penetrance fold change, including ARIH2, IL1A, RAD23B, TP53, EAPP, GAGE2, GAPDH, and $X A G E 2$ after an analysis comparing IE sera and non-IE control sera. In addition, we observed target antigens with high penetrance fold change, including $D B N L$, STAM2, ARIH2, CDK16, $B A G 4, N R I P 3$, and $R A D 23 B$ after an analysis comparing IE sera and healthy control sera. Third, the top 10 of identified antigens included BPI, IL1A, RAD23B, TP53, ARIH2, EAPP, 
Table S3. Proposed functional annotation of top 10 antigens from the Immunome ${ }^{\text {TM }}$ protein microarray platform with significant autoantibody responses in endocarditis patients' samples versus negative control

\begin{tabular}{llll}
\hline Rank $\quad$ Protein Symbol & Protein Name Uniprot Accession $\quad$ Proposed functional annotation
\end{tabular}

Interleukin-1 alpha

UV excision

repair protein

RAD23 homo-

$\log B$
P14778

P54727 omain-containing protein

094818
Receptor for IL1A, IL1B and IL1RN. After binding to interleukin-1 associates with the coreceptor IL1RAP to form the high affinity interleukin-1 receptor complex which mediates interleukin-1-dependent activation of NF-kappa-B, MAPK and other pathways. Signaling involves the recruitment of adapter molecules such as TOLLIP, MYD88, and IRAK1 or IRAK2 via the respective TIR domains of the receptor/coreceptor subunits. Binds ligands with comparable affinity and binding of antagonist IL1RN prevents association with IL1RAP to form a signaling complex. Involved in IL1B-mediated costimulation of IFNG production from T-helper 1 (Th1) cells.

Multiubiquitin chain receptor involved in modulation of proteasomal degradation. Binds to polyubiquitin chains. Proposed to be capable to bind simultaneously to the $26 \mathrm{~S}$ proteasome and to polyubiquitinated substrates and to deliver ubiquitinated proteins to the proteasome. May play a role in endoplasmic reticulum-associated degradation (ERAD) of misfolded glycoproteins by association with PNGase and delivering deglycosylated proteins to the proteasome.

Involved in global genome nucleotide excision repair (GG-NER) by acting as component of the XPC complex. Cooperatively with CETN2 appears to stabilize XPC. May protect XPC from proteasomal degradation.

The XPC complex is proposed to represent the first factor bound at the sites of DNA damage and together with other core recognition factors, XPA, RPA and the TFIIH complex, is part of the pre-incision (or initial recognition) complex. The XPC complex recognizes a wide spectrum of damaged DNA characterized by distortions of the DNA helix such as single-stranded loops, mismatched bubbles or single-stranded overhangs. The orientation of XPC complex binding appears to be crucial for inducing a productive NER. XPC complex is proposed to recognize and to interact with unpaired bases on the undamaged DNA strand which is followed by recruitment of the TFIIH complex and subsequent scanning for lesions in the opposite strand in a 5'-to-3' direction by the NER machinery. Cyclobutane pyrimidine dimers (CPDs) which are formed upon UV-induced DNA damage esacpe detection by the XPC complex due to a low degree of structural perurbation. Instead they are detected by the UV-DDB complex which in turn recruits and cooperates with the XPC complex in the respective DNA repair. In vitro, the XPC:RAD23B dimer is sufficient to initiate NER; it preferentially binds to cisplatin and UV-damaged double-stranded DNA and also binds to a variety of chemically and structurally diverse DNA adducts. XPC:RAD23B contacts DNA both $5^{\prime}$ and $3^{\prime}$ of a cisplatin lesion with a preference for the $5^{\prime}$ side. XPC:RAD23B induces a bend in DNA upon binding. XPC:RAD23B stimulates the activity of DNA glycosylases TDG and SMUG1.

RNA binding

Participates in the primary piRNA biogenesis pathway and is required during spermatogenesis to repress transposable elements and prevent their mobilization, which is essential for the germline integrity. The piRNA metabolic process mediates the repression of transposable elements during meiosis by forming complexes composed of piRNAs and Piwi proteins and govern the methylation and subsequent repression of transposons. Required for the final steps of primary piRNA biogenesis by participating in the processing of 31-37 nt intermediates into mature piRNAs. May act in pi-bodies and piP-bodies by transferring piRNA precursors or intermediates to or between these granules. 
Table S3. Proposed functional annotation of top 10 antigens from the Immunome $\mathrm{TM}^{\mathrm{T}}$ protein microarray platform with significant autoantibody responses in endocarditis patients' samples versus negative control [CONTINUED]

\begin{tabular}{|c|c|c|c|c|}
\hline 5 & $\mathrm{ARIH} 2$ & $\begin{array}{l}\text { E3 ubiquitin- } \\
\text { protein ligase } \\
\text { ARIH2 }\end{array}$ & O95376 & $\begin{array}{l}\text { E3 ubiquitin-protein ligase, which catalyzes ubiquitination of target proteins together } \\
\text { with ubiquitin-conjugating enzyme E2 UBE2L3 (PubMed:16118314, PubMed:17646546, } \\
\text { PubMed:19340006, PubMed:24076655). Acts as an atypical E3 ubiquitin-protein ligase } \\
\text { by working together with cullin-5-RING ubiquitin ligase complex (ECS complex, also } \\
\text { named CRL5 complex) and initiating ubiquitination of ECS substrates: associates with } \\
\text { ECS complex and specifically mediates addition of the first ubiquitin on ECS targets } \\
\text { (By similarity). The initial ubiquitin is then elongated (By similarity). E3 ubiquitin-pro- } \\
\text { tein ligase activity is activated upon binding to neddylated form of the ECS complex } \\
\text { (PubMed:24076655). Mediates 'Lys-6', 'Lys-48'-and 'Lys-63'-linked polyubiquitination } \\
\text { (PubMed:16118314, PubMed:17646546, PubMed:19340006). May play a role in myelo- } \\
\text { poiesis (PubMed:19340006). }\end{array}$ \\
\hline 6 & TP53 & $\begin{array}{l}\text { Cellular tumor } \\
\text { antigen p53 }\end{array}$ & Q96S44 & $\begin{array}{l}\text { Component of the EKC/KEOPS complex that is required for the formation of a thre- } \\
\text { onylcarbamoyl group on adenosine at position } 37 \text { (t6A37) in tRNAs that read codons } \\
\text { beginning with adenine (PubMed:22912744, PubMed:27903914). The complex is prob- } \\
\text { ably involved in the transfer of the threonylcarbamoyl moiety of threonylcarbamoyl- } \\
\text { AMP (TC-AMP) to the N6 group of A37 (PubMed:22912744, PubMed:27903914). } \\
\text { TP53RK has ATPase activity in the context of the EKC/KEOPS complex and likely } \\
\text { plays a supporting role to the catalytic subunit OSGEP (By similarity). Atypical protein } \\
\text { kinase that phosphorylates 'Ser-15' of p53/TP53 protein and may therefore partici- } \\
\text { pate in its activation (PubMed:11546806). }\end{array}$ \\
\hline 7 & EAPP & $\begin{array}{l}\text { E2F-associated } \\
\text { phosphoprotein }\end{array}$ & Q56P03 & $\begin{array}{l}\text { May play an important role in the fine-tuning of both major E2F1 activities, the regula- } \\
\text { tion of the cell-cycle and the induction of apoptosis. Promotes S-phase entry, and } \\
\text { inhibits p14(ARP) expression. }\end{array}$ \\
\hline 8 & GAGE2 & $\begin{array}{l}\mathrm{G} \text { antigen } \\
2 \mathrm{~B} / 2 \mathrm{C}\end{array}$ & Q13066 & $\begin{array}{l}\text { Antigen, recognized on melanoma by autologous cytolytic T-lymphocytes. } \\
\text { This gene belongs to a family of genes organized in clustered repeats. They have a } \\
\text { high degree of predicted sequence identity, but differ by scattered single nucleotide } \\
\text { substitution. Their sequences contain either the antigenic peptide YYWPRPRRY or } \\
\text { YRPRPRRY which is recognized by cytotoxic T-cells. }\end{array}$ \\
\hline 10 & XAGE2 & $\begin{array}{c}X \text { antigen family } \\
\text { member } 2\end{array}$ & Q96GT9 & highly expressed in normal testis \\
\hline
\end{tabular}

GAGE2, GAPDH, XAGE2, and ZNF174 after comparing the autoantibody profiles among IE, non-IE control, healthy control, and pooled normal samples. Fourth, STAM2, NRIP3, IL1A, RAD23B, CDK16, BAG4, DBNL, NRIP3, and ARIH2 were identified as the top 10 antigens throughout the analysis.
Finally, GAPDH and $D B N L$ were identified as clinically significant common diagnostic biomarkers for IE. However, further study to validate these novel biomarkers is warranted.

Generally, CRP or PCT cannot be used to diagnose IE in patients with bacteraemia. However, several potential 
Table S4. Proposed functional annotation of top 10 antigens from the Immunome ${ }^{\text {TM }}$ protein microarray platform with significant autoantibody responses in endocarditis patients' samples vs. healthy control

\begin{tabular}{|c|c|c|c|c|}
\hline Rank & Protein Symbol & Protein Name & Uniprot Accession & Proposed functional annotation \\
\hline
\end{tabular}
E3 ubiquitin-protein
ligase $A R I H 2$

UV excision repair protein RAD23 homolog B

\section{Cellular tumor} antigen $p 53$

E2F-associated phosphoprotein
E3 ubiquitin-protein ligase, which catalyzes ubiquitination of target proteins together with ubiquitin-conjugating enzyme E2 UBE2L3 (PubMed:16118314, PubMed:17646546, PubMed:19340006, PubMed:24076655). Acts as an atypical E3 ubiquitin-protein ligase by working together with cullin-5-RING ubiquitin ligase complex (ECS complex, also named CRL5 complex) and initiating ubiquitination of ECS substrates: associates with ECS complex and specifically mediates addition of the first ubiquitin on ECS targets (By similarity). The initial ubiquitin is then elongated (By similarity). E3 ubiquitin-protein ligase activity is activated upon binding to neddylated form of the ECS complex (PubMed:24076655). Mediates 'Lys-6', 'Lys-48'-and 'Lys-63'-linked polyubiquitination (PubMed:16118314, PubMed:17646546, PubMed:19340006). May play a role in myelopoiesis (PubMed:19340006)

Multiubiquitin chain receptor involved in modulation of proteasomal degradation. Binds to polyubiquitin chains. Proposed to be capable to bind simultaneously to the 265 proteasome and to polyubiquitinated substrates and to deliver ubiquitinated proteins to the proteasome. May play a role in endoplasmic reticulum-associated degradation (ERAD) of misfolded glycoproteins by association with PNGase and delivering deglycosylated proteins to the proteasome.

Involved in global genome nucleotide excision repair (GG-NER) by acting as component of the XPC complex. Cooperatively with CETN2 appears to stabilize XPC. May protect XPC from proteasomal degradation.

The XPC complex is proposed to represent the first factor bound at the sites of DNA damage and together with other core recognition factors, XPA, RPA and the TFIIH complex, is part of the pre-incision (or initial recognition) complex. The XPC complex recognizes a wide spectrum of damaged DNA characterized by distortions of the DNA helix such as single-stranded loops, mismatched bubbles or single-stranded overhangs. The orientation of XPC complex binding appears to be crucial for inducing a productive NER. XPC complex is proposed to recognize and to interact with unpaired bases on the undamaged DNA strand which is followed by recruitment of the TFIIH complex and subsequent scanning for lesions in the opposite strand in a 5'-to-3' direction by the NER machinery. Cyclobutane pyrimidine dimers (CPDs) which are formed upon UV-induced DNA damage esacpe detection by the XPC complex due to a low degree of structural perurbation. Instead they are detected by the UV-DDB complex which in turn recruits and cooperates with the XPC complex in the respective DNA repair. In vitro, the XPC:RAD23B dimer is sufficient to initiate NER; it preferentially binds to cisplatin and UV-damaged double-stranded DNA and also binds to a variety of chemically and structurally diverse DNA adducts. XPC:RAD23B contacts DNA both $5^{\prime}$ and $3^{\prime}$ of a cisplatin lesion with a preference for the $5^{\prime}$ side. XPC:RAD23B induces a bend in DNA upon binding. XPC:RAD23B stimulates the activity of DNA glycosylases TDG and SMUG1.

Component of the EKC/KEOPS complex that is required for the formation of a threonylcarbamoyl group on adenosine at position 37 (t6A37) in tRNAs that read codons beginning with adenine (PubMed:22912744, PubMed:27903914). The complex is probably involved in the transfer of the threonylcarbamoyl moiety of threonylcarbamoylAMP (TC-AMP) to the N6 group of A37 (PubMed:22912744, PubMed:27903914). TP53RK has ATPase activity in the context of the EKC/KEOPS complex and likely plays a supporting role to the catalytic subunit OSGEP (By similarity). Atypical protein kinase that phosphorylates 'Ser-15' of p53/TP53 protein and may therefore participate in its activation (PubMed:11546806).

May play an important role in the fine-tuning of both major E2F1 activities, the regulation of the cell-cycle and the induction of apoptosis. Promotes S-phase entry and inhibits p14(ARP) expression. 
Table S4. Proposed functional annotation of top 10 antigens from the Immunome ${ }^{T M}$ protein microarray platform with significant autoantibody responses in endocarditis patients' samples versus healthy control [CONTINUED]

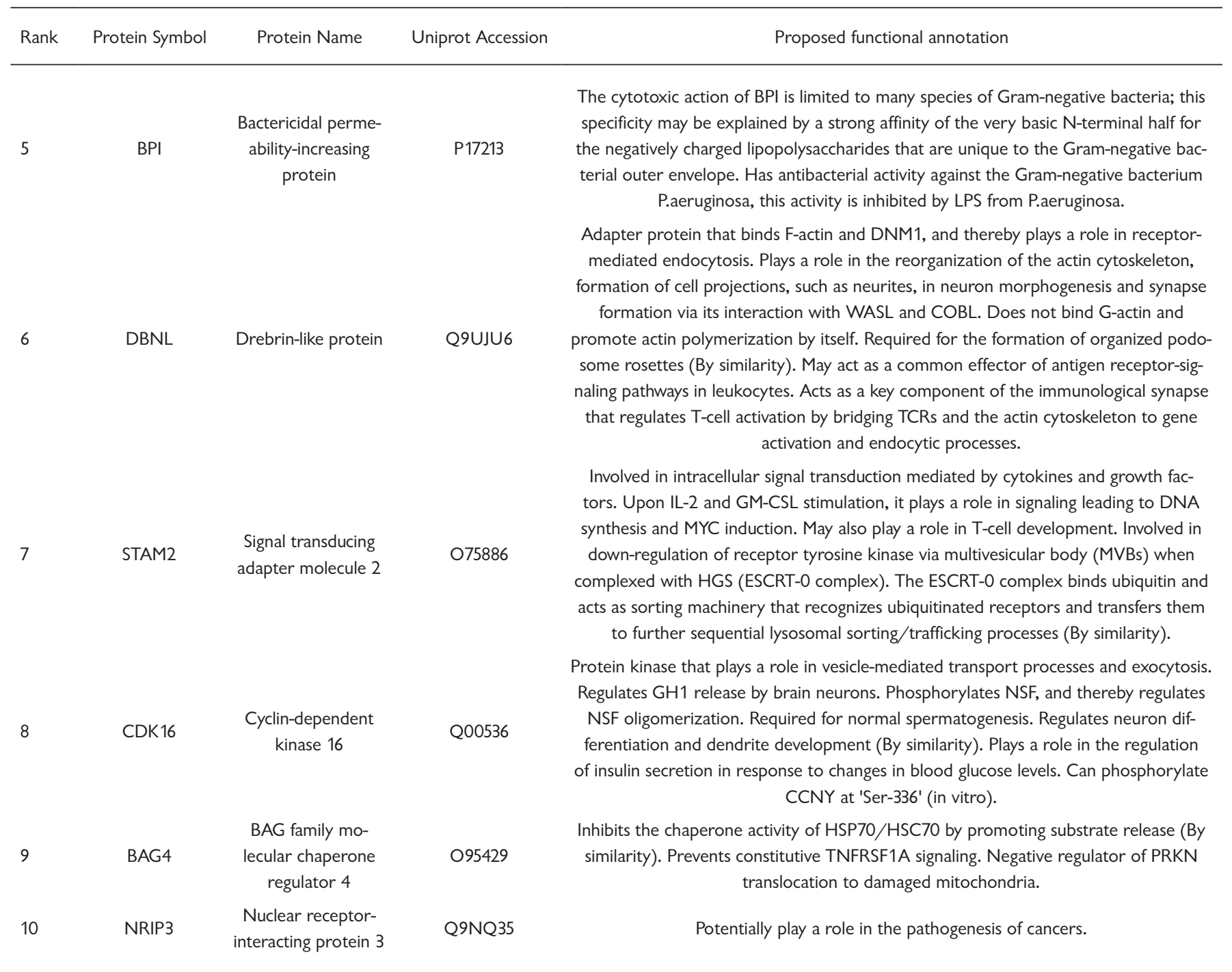

biomarkers have been reported, such as NT-proBNP, Cys C, LBP, troponins, $A Q P 9$, S100A11, E-selectin (CD62E), VCAM-1 (CD54), and IL-6 [Snipsoyr 2016]. Current study showed that previously reported potential biomarkers for IE, including ESR, CRP, RF, PCT, and NT-proBNP, were partially significant. Yet, these findings are important for identifying clinically significant common diagnostic biomarkers for IE, and the combination of biomarkers with diagnostic and prognostic potential of defined IE condition can improve the accuracy rate.

This study aimed to discover the most significant autoantibody biomarkers for IE. The proposed functional annotation of the top 10 antigens from the Immunome ${ }^{\mathrm{TM}}$ protein microarray platform with significant autoantibody responses in endocarditis patient versus non-IE control samples is listed in S3 Table, and the comparison of their annotations for endocarditis patient samples vs. healthy control samples is listed in S4 Table. From among the most significant autoantibody biomarkers listed for
IE, the following two biomarkers may have clinical relevance. First, GAPDH showed a penetrance fold change between IE patients and non-IE control as 5.667 and penetrance percentage frequency as $66.667 \%$. Surface-bound GAPDH has been found on the cells of all tested streptococcal species, in which it has been implicated as a virulence factor. In contrast, a soluble extracellular GAPDH was observed as the major secreted protein from S. gordonii FSS2, an endocarditis strain [Nelson 2001]. Streptococcus species are primary etiologic agents of IE, able to coordinate their gene expression through a quorum-sensing system, which assists their survival and regulates virulence [Teles 2012]. Antibiotics can modulate the expression of bacterial virulenceassociated genes, which can have counterproductive effects on the treatment of endocarditis [Yim 2006]. Indeed, it has been demonstrated that Streptococcus species has been shown to be with surface-bound $G A P D H$, where implicated as a virulence factor [Nelson 2001]. Increased plasminogen binding ability of 
streptococci from endocarditis isolates, when exposed to antibiotics, was due to an overexpression GAPDH [Teles 2012]. These results may clearly explain the mechanisms involved in the response of these organisms to the antimicrobials that can modulate the expression of bacterial genes of endocarditis.

Second is $D B N L$, with a penetrance fold change between IE patients and healthy control as 26.120 and penetrance percentage frequency as $33.333 \%$. The activation of $\beta$-adrenergic receptors ( $\beta$-ARs) plays a key role in regulating cardiac function [Liu 2014]. However, the detailed regulatory mechanisms of $\beta$-AR-induced fibrosis are still uncertain [Liu 2014]. $D B N L$ (also called HIP-55) was revealed as a novel regulator in the signalling regulatory network of $\beta$-AR activation [Liu 2014]. Further studies of both HIP-55-overexpressed and -deficient cardiac fibroblasts indicated that HIP-55 negatively regulated $\beta$-AR-activated cardiac fibroblast proliferation, and the proliferative signaling pathway may be associated with the extracellular signal-regulated protein kinase (ERK) activation [Liu 2014].

\section{CONCLUSIONS}

This study of five subjects screened across 1,600+ antigens on the Sengenics Immunome ${ }^{\mathrm{TM}}$ Protein Array and provides a general outlook on autoantibody biomarker profiles of IE patients. Findings indicate that GAPDH and DBNL particularly may serve as valuable diagnostic biomarkers. Therefore, further study, which may include a large-scale auto-antibody profiling study to identify biomarkers with diagnostic and prognostic potential of defined IE condition, is warranted to validate these novel biomarkers.

\section{ACKNOWLEDGEMENTS}

The authors wish to thank Lai Mei-Chun and Hong Ruei-Ping for sample collections. This research project would not have been possible without the support of many people. The authors wish to express their gratitude to the staffs of the Division of Cardiology, Division of Cardiovascular Surgery, Division of Critical Care Medicine, Division of Infectious Diseases, Department of Pharmacology, Department of Computer Science, and Department of Healthcare Quality at Changhua Christian Hospital. These individuals were extremely helpful and provided invaluable assistance and support. The authors also would like to thank AllBio Co. Ltd. (Taichung, Taiwan) and Sengenics Corporation Pte Ltd. (Singapore) for technical support. This manuscript was edited by Editage Editing.

\section{REFERENCES}

Beynon RP, Bahl VK, Prendergast BD. 2006. Infective endocarditis. BMJ 333:334-9.

Chen CH. 1997. Nosocomial Infective Endocarditis: Four Cases Report. J Infect Dis Soc ROC. 8:76-9.
Chen CH, Chang IL, Wang SH, et al. 2020. Potential Novel Proteomic Biomarkers for Diagnosis of Vertebral Osteomyelitis Identified Using an Immunomics Protein Array Technique: Two Cases Reports. Medicine (Baltimore) (revised).

Chen CH, Lo MC, Hwang K, et al. 2001. Infective endocarditis with neurologic complications: 10-year experience. J Microbiol Immunol Infect. 34:119-24.

Chen CH, Tsai CD, Wu SL, et al. 2001. A retrospective study of infective endocarditis at Changhua Christian Hospital during the years 19891999. J Intern Med Taiwan. 12:177-83.

Cornelissen CG, Frechen DA, et al. 2013. Inflammatory parameters and prediction of prognosis in infective endocarditis. BMC Infect Dis. 13:272.

Gu Z, Eils R, Schlesner M. 2016. Complex heatmaps reveal patterns and correlations in multidimensional genomic data. Bioinformatics (Oxford, England). 32:2847-9.

Habib G, Hoen B, Tornos PT, et al. 2009. Guidelines on the prevention, diagnosis, and treatment of infective endocarditis (new version 2009): the Task Force on the Prevention, Diagnosis, and Treatment of Infective Endocarditis of the European Society of Cardiology (ESC). Endorsed by the European Society of Clinical Microbiology and Infectious Diseases (ESCMID) and the International Society of Chemotherapy (ISC) for Infection and Cancer. Eur Heart J. 30:2369-413.

Habib G, Lancellotti P, Antunes MJ, et al. 2015.2015 ESC Guidelines for the management of infective endocarditis: The Task Force for the Management of Infective Endocarditis of the European Society of Cardiology (ESC). Endorsed by: European Association for Cardio-Thoracic Surgery (EACTS), the European Association of Nuclear Medicine (EANM). Eur Heart J. 36:3075-128.

Knudsen JB, Fuursted K, Petersen E, et al. 2009. Infective endocarditis: a continuous challenge. The recent experience of a European tertiary center. J Heart Valve Dis.18:386-94.

Kocazeybek B, Kucukoglu S, Oner YA. 2003. Procalcitonin and C-reactive protein in infective endocarditis: correlation with etiology and prognosis. Chemotherapy. 49:76-84.

Koscielny G, An P, Carvalho-Silva D, et al. 2017. Open Targets: a platform for therapeutic target identification and validation. Nucleic Acids Res. 45:D985-D94.

Lang RM, Bierig M, Devereux RB, et al. 2005. Recommendations for chamber quantification: a report from the American Society of Echocardiography's Guidelines and Standards Committee and the Chamber Quantification Writing Group, developed in conjunction with the European Association of Echocardiography, a branch of the European Society of Cardiology. J Am Soc Echocardiogr. 18:1440-63.

Li JS, Sexton DJ, Mick N, et al. 2000. Proposed modifications to the Duke criteria for the diagnosis of infective endocarditis. Clin Infect Dis 30:633-8.

Liu N, Xing R, Yang C, et al. 2014. HIP-55/DBNL-dependent regulation of adrenergic receptor mediates the ERK1/2 proliferative pathway. Molecular bioSystems. 10:1932-9.

Nelson D, Goldstein JM, Boatright K, et al. 2001. pH-regulated secretion of a glyceraldehyde-3-phosphate dehydrogenase from Streptococcus gordonii FSS2: purification, characterization, and cloning of the gene encoding this enzyme. J Dent Res. 80:371-7.

Nishimura RA, Otto CM, Bonow RO, et al. 2014. 2014 AHA/ACC guideline for the management of patients with valvular heart disease: executive summary: a report of the American College of Cardiology/ 
American Heart Association Task Force on Practice Guidelines. J Am Coll Cardiol. 63:2438-88.

Snipsoyr MG, Ludvigsen M, Petersen E, et al. 2016. A systematic review of biomarkers in the diagnosis of infective endocarditis. Int J Cardiol. 202:564-70.

Teles C, Smith A, Lang S. 2012. Antibiotic modulation of the plasminogen binding ability of viridans group streptococci. Antimicrob Agents Chemother. 56:458-63.

Thiene G, Basso C. 2006. Pathology and pathogenesis of infective endocarditis in native heart valves. Cardiovasc Pathol. 15, 256-263.

Ward Jr JH. 1965. Hierarchical grouping to optimize an objective function J Am Stat Assoc. 58:236-44.
Watkin RW, Harper LV, Vernallis AB, et al. 2007. Pro-inflammatory cytokines IL6, TNF-alpha, IL1beta, procalcitonin, lipopolysaccharide binding protein and C-reactive protein in infective endocarditis. J Infect. 55:220-5.

Wei XB, Chen F, Huang JL, et al. 2017. Novel Risk Biomarker for Infective Endocarditis Patients With Normal Left Ventricular Ejection Fraction-Monocyte to High-Density Lipoprotein Cholesterol Ratio. Circ J. 82, 283-288.

Yim G, Wang HH, Davies J. 2006. The truth about antibiotics. Int J Med Microbiol. 296:163-70. 